\title{
A Low-Complexity Joint TOAs and AOAs Parameter Estimator Using Dimension Reduction for FMCW Radar Systems
}

\author{
Sangdong Kim, Yeonghwan Ju, Jonghun Lee \\ Advanced Radar Technology Laboratory, Convergence Research Center for Future Automotive \\ Technology, Daegu Gyeongbuk Institute of Science \& Technology, \\ Daegu, Republic of Korea \\ jhlee@dgist.ac.kr
}

\begin{abstract}
We propose a low-complexity of joint time delay of arrivals (TOAs) and angles of arrivals (AOAs) using dimension reduction for frequency modulated continuous-wave (FMCW) radar systems. In the road environment, the FMCW radar can obtain the position of other vehicles accurately since the radar system is able to estimate spatial-temporal parameters such as TOAs and AOAs of multiple targets. The requirement of $\mathrm{FMCW}$ radar is increasing in terms of the parameter accuracy and resolution over time. In order to accomplish high accuracy and resolution of the parameters such as range, velocity, and angle, conventional two dimensional- discrete Fourier transform (2D-DFT) have reduced performance of the parameter estimation. To enhance the estimation performance of parameter, proposed high resolution based methods, which are two dimensional-multiple signal classification (2D-MUSIC), is utilized. However, conventional high-resolution methods are not used in real-time systems due to their high complexity situation. In order to meet resolution ability and automotive radar system requirements, we present a joint TOAs and AOAs parameter estimator using dimension reduction with combination of the DFT and the MUSIC algorithm for low-complexity FMCW radar systems. In experimental results of multi-target environments, in terms of the estimation performance, the proposed method is better than 2D-MUSIC.
\end{abstract}

Index Terms-Joint delay and angle; 2D-MUSIC; Low complexity; Dimension reduction; DFT-MUSIC.

\section{INTRODUCTION}

Spatial-temporal parameters such as multipath time delays of arrivals (TOAs) and angles of arrivals (AOAs) have been widely studied for radar systems and wireless communication systems [1]. These two parameters are useful for wireless communication and localization of moving targets in radar systems. For a very long time, frequency modulated continuous-wave (FMCW) radar systems are widely utilized for a variety of fields in order to obtain the TOAs and AOAs of moving targets. Especially, phase array beamforming based on vehicle radar sensor systems [2] is used for smart cruise control, traffic monitoring and collision avoidance [3] [6] and airborne radar is utilized civil and military applications with high-resolution detection and small size

Manuscript received 22 October, 2017; accepted 16 May, 2018.

This work was supported by the DGIST R\&D Program of the Ministry of Science, ICT and Future Planning, Korea (18-IT-01). system [7]-[11]. By a de-chirping method of an FMCW radio frequency (RF) module, the received signals are able to be transformed into sinusoidal waveforms in order to acquire TOAs and AOAs information. We can define these sinusoidal signals as beat signals. The de-chirping system simplifies the radar hardware, especially for digital signal processing, because the bandwidth of the beat signal is reduced as compared with FMCW transmitted (TX) signals. For this reason, interest in FMCW radar systems has risen considerably along with the need for the estimation of the joint TOAs and AOAs of targets.

The parameter accuracy and resolution of the FMCW radar should be improved because automotive application demands more high requirements over time. In terms of one dimensional parameter estimator, from conventional discrete Fourier transform (DFT) [3] to multiple signal classification (MUSIC) [6], a variety of estimation algorithms have been utilized for automotive radar. However, one dimensional parameter estimator cannot obtain the multiple parameters jointly. Hence, we need to consider two dimensional parameter estimator.

In case of two dimensional parameter estimator, conventional two dimensional-DFT (2D-DFT) suffers from performance degradation to obtain high precision and resolution of parameters such as range, speed and angle. In order to enhance the estimation performance of joint TOA and AOA parameters, conventional 2D algorithms for high-resolution, which have 2D-ESPRIT (two dimensional-estimation of signal parameters via rotational invariant technique) [12] and two dimensional-MUSIC (2D-MUSIC) [13], have been used to estimate parameters of multiple targets. However, real-time radar systems cannot use conventional high-resolution algorithms due to their high computational burden.

For this context, various low complexity 2D high-resolution algorithms have been proposed lately. In [14], this paper proposed a low complexity ESPRIT based DOA estimator for collocated MIMO radar. To order to reduce complexity, they use the property that the Kronecker product results of transmit and receive steering vectors can be implemented as one new steering vector. However, this paper has limitations that can only be used to estimate DOA parameters for specific condition as MIMO radar. In [15], 
this paper proposed an algorithm that can find noise subspace based on the cross correlation function of received signal without SVD or EVD. However, this method has a disadvantage that it cannot be used for a single Uniform Linear Array (ULA) such as vehicle radar because it is based on the cross correlation function between the received data collected by the two component ULAs of L-shaped array. Therefore, conventional low complexity 2D high-resolution algorithms still have high complexity, which is difficult to apply to vehicle radar systems, and there are limitations that can only operate in certain conditions such as L-shaped.

Therefore, we propose a low-complexity of joint TOAs and AOAs using dimension reduction (DR) with combination of the DFT and the MUSIC algorithm for FMCW radar systems. This paper is composed as follows. Section II explains the system model. In Section III, we analyse the structure of the conventional 2D-MUSIC estimator. In Section IV, we propose the low-complexity of joint TOAs and AOAs using DR with combination of the DFT and the MUSIC algorithm for FMCW radar systems. In Section V, we conduct a simulation to assess the estimation results of the proposed algorithm. In Section VI, we measure the CPU execution time in MATLAB to evaluate the complexity reduction of the proposed algorithm. In Section VII, we assess the estimation performance of the proposed method through real experiments. Finally, we conclude the paper in Section VIII.

Notation: $(.)^{\mathrm{T}}$ and $(.)^{\mathrm{H}}$ denote transpose and conjugate transpose, respectively. Operator $\otimes$ is the Kronecker product and $\boldsymbol{I}_{L}$ is the $L \times L$ identity matrix.

\section{SignAl MODEL}

In the time domain, the TX FMCW signal $s(t)$ is expressed as

$$
s(t)=\exp \left(j\left(2 \pi f_{s} t+\mu t^{2} / 2\right)\right) \text { for } 0 \leq t<T_{\mathrm{sym}},
$$

where $f_{s}$ is the initial frequency, $\mu$ is the rate of change of the instantaneous frequency of a chirp symbol and $T_{\text {sym }}$ is the signal period of a FMCW symbol.

For a ULA consisting of $K$ elements as shown in Fig. 1, we consider $M$ targets. Over $T_{\text {sym }}$ for the $m$-th target, we can represent the reflected signal in a time-invariant enviroment at $k$-th antenna element with the complex amplitude $a_{m}$ in [12] such that

$$
y_{k}(t)=\sum_{m=0}^{M-1} a_{m} s\left(t-\tau_{m}\right) \exp \left(j \frac{2 \pi}{\lambda} d k \sin \theta_{m}\right)+\omega_{k}(t),
$$

where $\tau_{m}$ is the TOA, $\lambda$ is the carrier frequency's wavelength, $d$ denotes the distance from $k$-th to $(k+1)$-th antenna element, $\theta_{m}$ defines the AOA and $\omega_{k}(t)$ means the $k$-th antenna position's additive white Gaussian noise (AWGN).

Using the reflected signal from targets, by de-chirping, the received FMCW signals $y(t)$ is able to multiply with the conjugation of the FMCW transmitted signals $s^{*}(t)$ such as

$$
d_{k}(t)=y_{k}^{*}(t) s(t)
$$

where $d_{k}(t)$ means $k$-th antenna array's output results of de-chirping method. In case of $d=\lambda / 2$, the transformed signals $d_{k}(t)$ in (3) can be expressed as a sinusoidal signal by

$$
\begin{gathered}
d_{k}(t)=\sum_{m=0}^{M-1} a_{m} \exp \left(j\left(\mu \tau_{m} t+\omega_{s} \tau_{m}-\frac{\mu}{2} \tau_{m}^{2}\right)\right) \times \\
\quad \times \exp \left(j \pi k \sin \theta_{m}\right)+\omega_{k}(t) .
\end{gathered}
$$

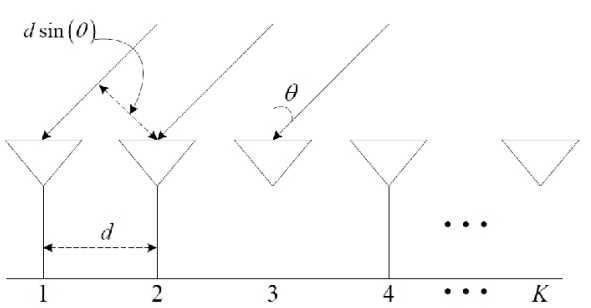

Fig. 1. The structure of ULA.

For Nyquist condition from an analog-to-digital converter (ADC), sampling frequency is set to $f_{s}=1 / T_{s}$, the digital received signal $d_{k}\left[n_{s}\right]$ of $d_{k}(t)$ can be represented such that

$$
\begin{gathered}
d_{k}\left[n_{s}\right]= \\
=\sum_{m=0}^{M-1} a_{m} \exp \left(j \left(\mu \tau_{m} T_{s} n_{s}+\omega_{s} \tau_{m}-\right.\right. \\
\left.\left.-\frac{\mu}{2} \tau_{m}{ }^{2}+\pi k \sin \theta_{m}\right)\right)+\omega_{k}\left[n_{s}\right],
\end{gathered}
$$

where $n_{s}=0,1, \ldots N-1$.

\section{CONVENTIONAL 2D-MUSIC EstimatoR}

The conventional 2D-MUSIC estimation algorithm is based on a stacking received data vector such that

$$
\mathbf{D}_{n}=\left[\mathbf{D}_{n, 0}, \mathbf{D}_{n, 1}, \ldots, \mathbf{D}_{n, K-1}\right]^{\mathrm{T}},
$$

where $\quad \mathbf{D}_{n, k}=\left[d_{k}[n], d_{k}[n+1], \ldots d_{k}[n+L-1]\right]^{\mathrm{T}} \quad$ and $\quad L$ means the parameter of selection for $2 \leq L<N$. Using the stacking received data matrix, the spatial-temporal covariance matrix can be estimated as $\mathbf{R}=1 /(N-L) \sum_{n=0}^{N-L-1} \mathbf{D}_{n} \mathbf{D}_{n}{ }^{\mathrm{H}} . \quad$ Through eigenvalue decomposition (EVD), the signal subspace matrix $\boldsymbol{U}_{\mathrm{s}}$ is the eigenvectors corresponding to the $M$ largest eigenvalues of $\boldsymbol{R}$. The noise subspace matrix $\boldsymbol{U}_{\mathrm{n}}$ is the eigenvectors corresponding to the $K L-M$ smallest eigenvalues of $\boldsymbol{R}$. Using the noise subspace matrix $\boldsymbol{U}_{\mathrm{n}}$, we can establish the 2D-MUSIC TOAs and AOAs spectrum function such as

$$
=\frac{f_{2 \mathrm{D}-\operatorname{MUSIC}}(\tau, \theta)=}{[a(\tau) \otimes a(\theta)]^{\mathrm{H}} \mathbf{U}_{\mathrm{n}} \mathbf{U}_{\mathrm{n}}{ }^{\mathrm{H}}[a(\tau) \otimes a(\theta)]},
$$

where $a(\tau)$ and $a(\theta)$ are TOA and AOA steering vectors, respectively, and they are expressed as:

$$
a(\tau)=\left[1, \exp \left(-j \mu \tau T_{S}\right), \ldots, \exp \left(-j \mu \tau T_{S}(N-1)\right)\right]^{\mathrm{T}},
$$


$a(\theta)=[1, \exp (-j \pi \sin \theta), \ldots, \exp (-j(K-1) \pi \sin \theta)]^{\mathrm{T}}$.

Here, we have the $M$ largest peaks of $f_{2 \mathrm{D}-\operatorname{MUsIC}}(\tau, \theta)$ taken as the estimates of the TOAs and AOAs for the automotive targets. For 2D-MUSIC, since a huge 2D search and EVD are needed, it is inefficient with high computational cost. In the following sections, we present the proposed low-complexity of joint TOAs and AOAs using DR with combination of the DFT and the MUSIC algorithm for FMCW radar systems

\section{PRoposed JoInt TOAS AND AOAs USING DR AND COMBINATION OF DFT AND MUSIC ALGORITHM}

The proposed DFT-MUSIC estimation algorithm is based on the stacking received data matrix of (6). The signal subspace matrix $\boldsymbol{U}_{\mathrm{s}}$ and the noise subspace matrix $\boldsymbol{U}_{\mathrm{n}}$ can separate using the EVD of $\boldsymbol{R}$. The noise subspace matrix $\boldsymbol{U}_{\mathrm{n}}$ is orthogonal to the actual target directional vector $\boldsymbol{P}$, i.e., $\left\|\mathbf{U}_{n} \mathbf{P}\right\|^{2}=0$ and $\boldsymbol{P}$ is denoted as:

$$
\mathbf{P}=\left[\begin{array}{c}
1 \\
\exp j\left(\mu \tau_{m} T_{s}\right) \\
\vdots \\
\exp j\left(\mu \tau_{m} T_{s}(L-1)\right) \\
\exp j\left(\pi \sin \theta_{m}\right) \\
\exp j\left(\mu \tau_{m} T_{s}+\pi \sin \theta_{m}\right) \\
\vdots \\
\exp j\left(\mu \tau_{m} T_{s}(L-1)+\pi \sin \theta_{m}\right) \\
\vdots \\
\exp \left(j\left(\mu \tau_{m} T_{s}(L-1)+\pi(K-1) \sin \theta_{m}\right)\right)
\end{array}\right]
$$

$\left\|\mathbf{U}_{n} \mathbf{P}\right\|^{2}$ can be rewritten as

$$
\left\|\mathbf{U}_{\mathrm{n}} \mathbf{P}\right\|^{2}=\mathbf{S}^{\mathrm{H}} \mathbf{T}^{\mathrm{H}} \mathbf{U}_{\mathrm{n}} \mathbf{U}_{\mathrm{n}}{ }^{\mathrm{H}} \mathbf{T S}=0,
$$

where

$$
\begin{gathered}
\mathbf{S}=\left[1, \exp \left(j \pi \sin \theta_{m}\right), \ldots, \exp \left(j \pi(K-1) \sin \theta_{m}\right)\right]^{\mathrm{T}}, \\
\mathbf{T}=\left[1, \exp \left(j \mu \tau_{m} T_{s}\right), \ldots, \exp \left(j \mu \tau_{m} T_{S}(L-1)\right)\right]^{\mathrm{T}} \otimes \mathbf{I}_{L} .
\end{gathered}
$$

We can transform the two dimension to one dimension through DR, which can separate TOA and the AOA parameters, by using (11). We first determine the TOAs by the DFT results of the received signal, as in [16]. In the 1-th antenna element, the indices of DFT of the received signal estimates the TOAs for multiple targets. We denote the $L$-point DFT such as

$$
\mathbf{D}_{\mathrm{DFT}}=\mathbf{D}_{n, 0}^{\mathrm{T}} \mathbf{F}_{L},
$$

where $\boldsymbol{F}$ is $L$-point DFT matrix i.e., $\boldsymbol{F}=\left[F_{1}, F_{2}, \ldots, F_{P}\right]$, which is $F_{\mathrm{p}}=[1, \exp (-j 2 \pi p / L), \ldots, \exp (-j 2 \pi(L-1) p / L)]^{\mathrm{T}}$, is the steering vector for the TOAs. After the DFT in the $1 \mathrm{st}$ antenna element and the peak finding are accomplished among the multiple targets, the TOA index vector is obtained,
$\boldsymbol{I}=\left[I_{0}, I_{1}, \ldots, I_{M-1}\right]$, where $I_{m}$ denotes $\mu \tau_{m} T_{s} L$. Therefore, the estimated TOA direction vector for the $m$-th target can be written as

$$
\begin{gathered}
\mathbf{T}\left(\hat{\tau}_{m}\right)= \\
=\left[1, \exp \left(j \mu \hat{\tau}_{m} T_{s}\right), \ldots, \exp \left(j \mu \hat{\tau}_{m} T_{s}(L-1)\right)\right]^{\mathrm{T}} \otimes \mathbf{I}_{L} .
\end{gathered}
$$

For each target AOA, by substituting the above estimated vector in (11), a normal MUSIC estimator at the $m$-th target is obtained such that

$$
f_{m}(\theta)=\frac{1}{\mathbf{S}(\theta)^{H} \mathbf{Q}_{m} \mathbf{S}(\theta)}
$$

where $\mathbf{Q}_{m}=\mathbf{T}\left(\hat{\tau}_{m}\right)^{H} \mathbf{U}_{\mathrm{n}} \mathbf{U}_{\mathrm{n}}{ }^{H} \mathbf{T}\left(\hat{\tau}_{m}\right)$. After the MUSIC estimation of the $m$-th target and peak detection processing, through the AOA index matrix $\boldsymbol{J}=\left[J_{0}, J_{1}, \ldots, J_{M-1}\right]$ of the multiple targets, the AOA of $m$-th target is accomplished by

$$
\hat{\theta}_{m}=\sin ^{-1}\left(\frac{2 J_{m}}{K}\right),
$$

where $\sin ^{-1}(\cdot)$ is the inverse operator of the sine function.

Thus, we have outlined the proposed DFT-MUSIC algorithm in order to TOAs and AOAs information for vehicle FMCW radar, as shown in Fig. 2.

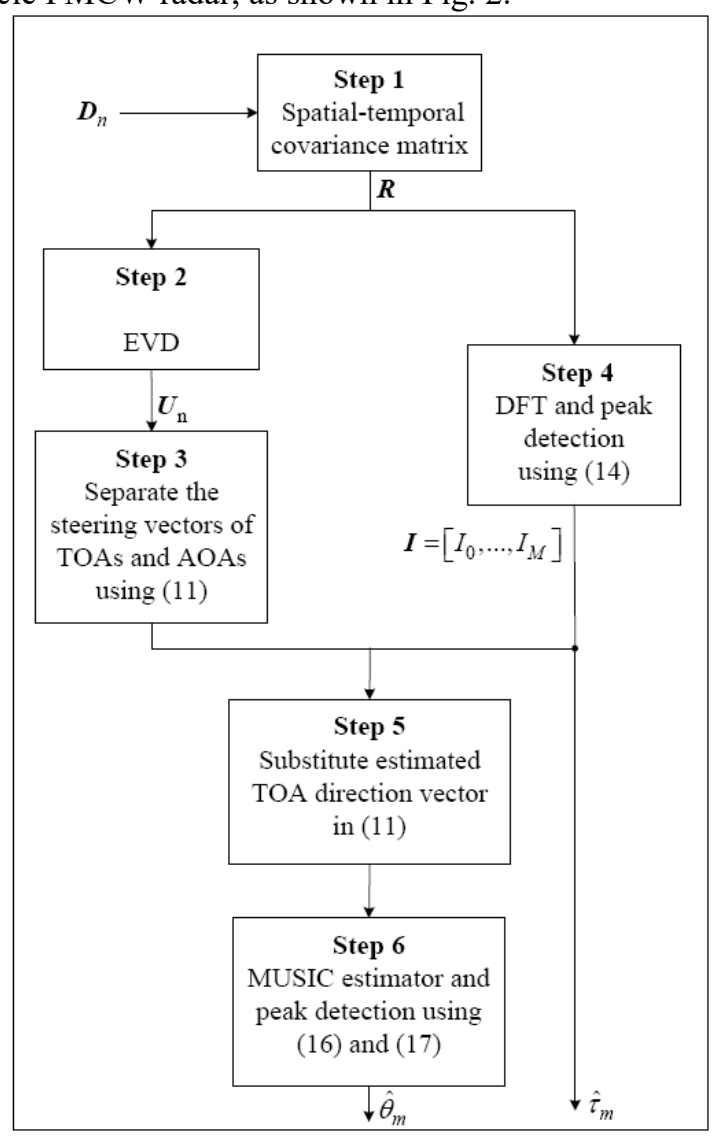

Fig. 2. Block diagram of the Joint DFT-MUSIC based proposed algorithm.

The major steps of the proposed algorithm are as follows:

Step 1: Construct the spatial-temporal covariance matrix $\boldsymbol{R}$.

Step 2: Through the EVD of $\boldsymbol{R}$, the signal subspace matrix 
$\boldsymbol{U}_{\mathrm{s}}$ and the noise subspace matrix $\boldsymbol{U}_{\mathrm{n}}$ are obtained, respectively.

Step 3: Through (11), separate the steering vectors of TOAs and the AOAs, respectively using DR.

Step 4: Search $\tau_{m}$ through the DFT and peak detection of DFT output, we can obtain the $M$ estimated TOA terms of multiple targets, i.e., $\hat{\tau}_{m}$ for $1 \leq m \leq M$.

Step 5: Substitute the estimated TOA direction vector of (15) in (11).

Step 6: Searching $\theta_{m}$ through the MUSIC estimator of (16) and peak detection, we can find the $M$ estimated AOA terms of multiple targets, i.e., $\hat{\theta}_{m}$ for $1 \leq m \leq M$.

\section{Simulation RESUltS AND COMPLEXITY ANALYSIS}

We conducted a simulation in MATLAB to assess the estimation results of the proposed algorithm. A snapshot of the time delay-angle map is obtained. In the section, the parameters are set as shown in Table I. We assume that a $24 \mathrm{GHz}$ RF module of a FMCW radar with one transmitting channel and two receiving channels is used in order to obtain the simulation results.

TABLE I. SIMULATION PARAMETERS FOR AWGN CHANNEL.

\begin{tabular}{|c|c|}
\hline Parameter & Value \\
\hline Change rate of chirp, $\mu$ & $3.125 \times 1012$ \\
\hline Sampling interval, Ts & $200 \mathrm{~ns}$ \\
\hline Symbol duration, Tsym & $80 \mu \mathrm{s}$ \\
\hline
\end{tabular}

For the simulation, as presented in Fig. 3, a single target was placed at $\left[R_{1}, \theta_{1}\right]=\left[6.75 \mathrm{~m}, 20^{\circ}\right]$. As in Fig. 3, the proposed algorithm and the conventional algorithm can correctly obtain similar angle and time delay results.

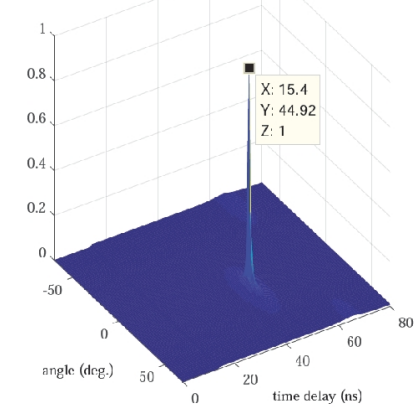

(a)

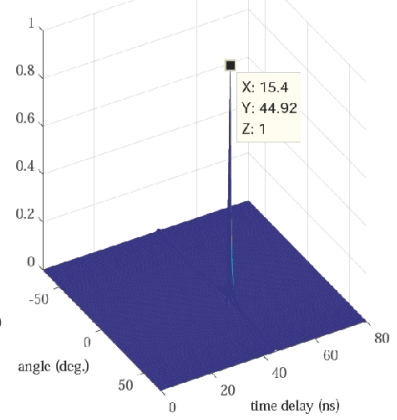

(b)
Fig. 3. Time delay-angle map results with $K=2$ for single target: a) conventional 2D-MUSIC; b) proposed.

Figure 4 shows simulation snapshot of two targets in an AWGN channel. Two different targets were placed at $\left[R_{1}\right.$, $\left.\theta_{1}\right]=\left[5.25 \mathrm{~m}, 10^{\circ}\right]$ and $\left[R_{2}, \theta_{2}\right]=\left[6.75 \mathrm{~m}, 20^{\circ}\right]$. The proposed algorithm and the conventional algorithm obtain similar angles and time delays results correctly.

In order to evaluate the complexity reduction by the proposed algorithm, we measured the CPU execution time in MATLAB. Figure 5 shows the measured CPU time according to the number of time samples $(L)$. The complexity of the proposed method is at least 100 times lower than that of the conventional 2D-MUSIC algorithm with $K=2$. It implies that the proposed scheme significantly reduces the computation complexity by employing dimension reduction compared to the conventional 2D MUSIC algorithm while achieving similar performance of 2D MUSIC algorithm.

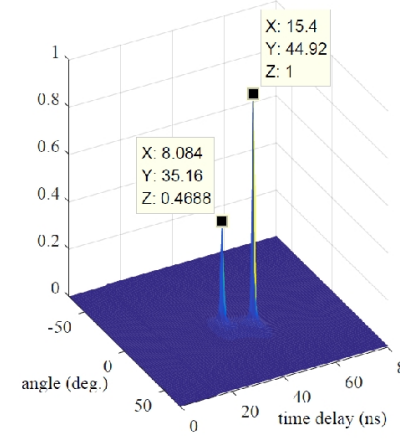

(a)

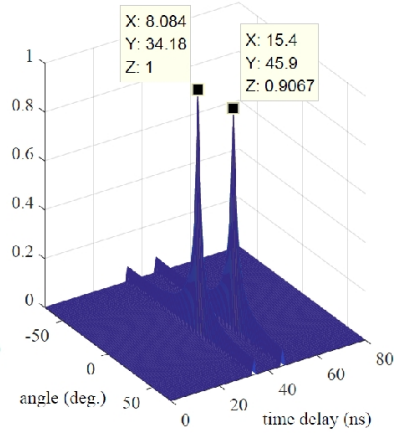

(b)
Fig. 4. Time delay-angle map results with $K=2$ for two targets: a) conventional 2D-MUSIC; b) proposed.

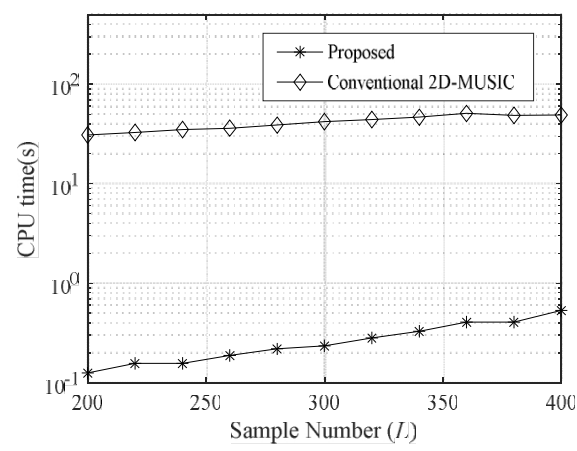

Fig. 5. MATLAB CPU execution time according to the number of samples $L$ for the conventional 2D MUSIC and the proposed DFT-MUSIC with $K=$ 2.

\section{EXPERIMENTS}

In various experiments, the evaluation of the estimation performance of the proposed method were carried out in an anechoic chamber located at DGIST in South Korea. With $24 \mathrm{GHz}$ carrier frequency, we produced a FMCW RF module with 1 TX channel and 2 received (RX) channels. The transmitter includes a frequency synthesizer, a voltage-controlled oscillator (VCO) and an oscillator with $26 \mathrm{MHz}$.

An FMCW signal is generated with the frequency synthesizer for the $200 \mathrm{MHz}$ bandwidth in the range of $24.05 \mathrm{GHz}-24.25 \mathrm{GHz}$. The receiver consists of three high-pass filters, three low-pass filters, three low noise amplifiers (LNAs) and three mixers. The overall noise figure of the receiver and noise figure of the LNA are set to $8 \mathrm{~dB}$ and $14 \mathrm{~dB}$, respectively. The gain was $2.5 \mathrm{~dB}$. By the mixer, the $\mathrm{RF}$ signal was changed to an intermediate frequency (IF) signal (beat signal). A photograph of the experimental chamber environment and the developed $24 \mathrm{GHz}$ FMCW RF module is shown in Fig. 6.

Figure 7 shows photo of experimental environment. In order to avoid the effect due to undesired reflected signal, the experiment is conducted in anechoic Chamber. For the experiments, a single target was placed at $\left[R_{1}, \theta_{1}\right]=[7.2 \mathrm{~m}$, $0^{\circ}$.

The experimental results of the time delay-angle map were estimated, as given in Fig. 8. As shown in Fig. 8, the proposed method and the conventional method estimated similar angles and time delays correctly, but the proposed algorithm had a lower complexity load than 2D-MUSIC. 


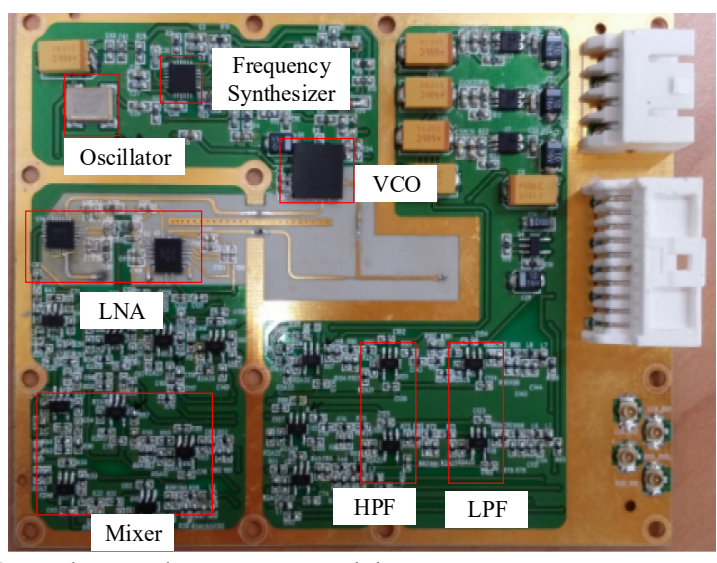

Fig. 6. Implemented FMCW RF module.

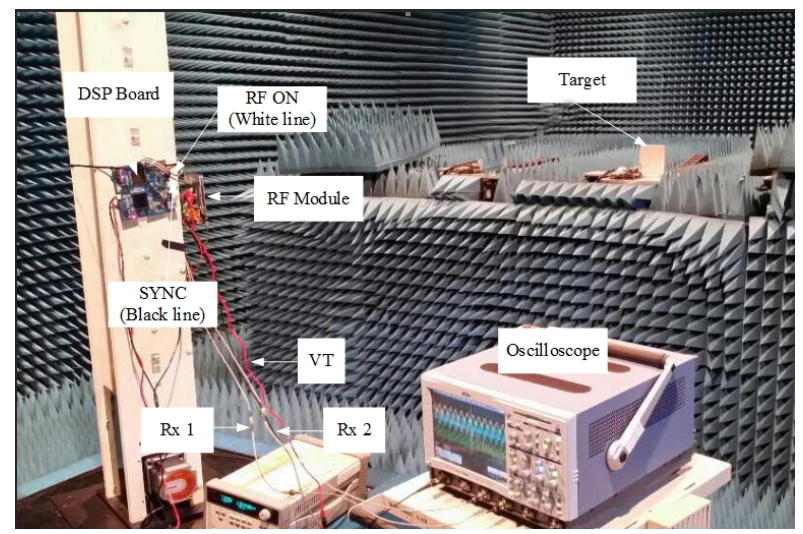

Fig. 7. Photo of experimental environment.

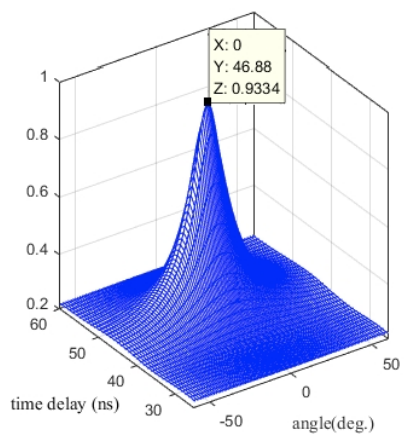

(a)

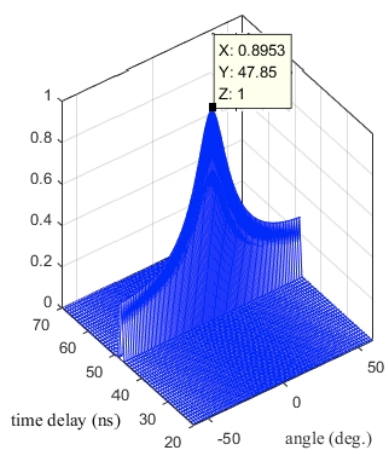

(b)
Fig. 8. The experimental results based on various algorithms: a) conventional 2D-MUSIC; b) proposed.

\section{CONCLUSIONS}

In this paper, we proposed a low-complexity of joint TOAs and AOAs using DR for FMCW radar systems. The FMCW radar for vehicles is suitable to solve the issues of complicated location because it can estimate the spatial-temporal parameters such as TOAs and AOAs of multiple targets. However, for the estimation of complicated locations, conventional methods with high-resolution such as 2D-MUSIC are not used in real-time systems because of their high complexity burden. In order to meet resolution ability and automotive radar system requirements, we present a joint time delay and angle parameter estimator using DR with the combination of the DFT and the MUSIC algorithm for low-complexity FMCW radar systems. To assess the proposed method, we conducted experiments using our developed $24 \mathrm{GHz} 2$ channel FMCW radar and measured the processing time by the CPU execution time. The processing time of the proposed method is at least 100 times lower than that of the conventional 2D-MUSIC algorithm with ULA arrays $K=2$. The results confirmed that the proposed method has a relatively lower complexity while the estimation performance of the proposed one is similar as that of the conventional method.

\section{REFERENCES}

[1] C. Lin, W. Fang, "Joint angle and delay estimation in frequency hopping systems", IEEE Trans. Aerosp. Electron. Syst., vol. 49, no. 2, pp. 1042-1056, 2013. DOI: 10.1109/TAES.2013.6494398.

[2] C. Schroeder, H. Rohling, "X-Band FMCW radar system with variable chirp duration", in Proc. of IEEE Radar Conf., 2010, pp. 1255-1259. DOI: 10.1109/RADAR.2010.5494425.

[3] S. Kim, D. Oh, J. Lee, "Joint DFT-ESPRIT estimation for TOA and DOA in vehicle FMCW radars", IEEE Antennas Wireless Propag. Lett., vol. 14, pp. 1710-1713, 2015. DOI: 10.1109/LAWP.2015.2420579.

[4] A. Townley, P. Swirhun, D. Titz, A. Bisognin, F. Gianesello, R. Pilard, C. Luxey, A. M. Niknejad, "A 94-GHz 4TX-4RX phased-array FMCW radar transceiver with antenna-in-package”, IEEE J. Solid-State Circuits, vol. 52, no. 5, pp. 1245-1259, 2017. DOI: 10.1109/JSSC.2017.2675907.

[5] S. Lee, Y. Yoon, J. Lee, S. Kim, "Human-vehicle classification using feature-based SVM in 77-GHz automotive FMCW radar", IET Radar Sonar Nav., vol. 11, no. 10, pp. 1589-1596, 2017. DOI: 10.1049/iet-rsn.2017.0126.

[6] V. K. Nguyen, M. D. E. Turley, G. A. Fabrizio, "A new data extrapolation approach based on spectral partitioning", IEEE signal Proess. Lett., vol. 23, no. 4, pp. 454-458, 2016. DOI: 10.1109/LSP.2016.2533602.

[7] A. Ganis, E. Navarro, B. Schoenlinner, U. Prechtel, A. Meusling, C. Heller, T. Spreng, J. Mietzner, C. Krimmer, B. Haeberle, S. Lutz, M. Loghi, A. Belenguer, H. Esteban, V. Ziegler, "A portable 3-D imaging FMCW MIMO radar demonstrator with a $24 \times 24$ antenna array for medium-range application", IEEE Tran. Geosci. Remote Sens., vol. 55, no. 2, pp. 834-843, 2017. DOI: 10.1109/TGRS.2017.2746739.

[8] J. Suh, L. Minz, D. Jung, H. Kang, J. Ham, S. Park, "Drone-based external calibration of a fully synchronized Ku-band heterodyne FMCW radar", IEEE Trans. Instrum. Meas., vol. 66, no. 8, pp. 21892197, 2017. DOI: 10.1109/TIM.2017.2687518.

[9] J. B. Yan, D. Gomez-Garcia, J. W. McDaniel, Y. Li, P. Gogineni, F. Rodriguez-Morales, J. Brozena, C. Leuschen, "Ultrawideband FMCW radar for airborne measurements of snow over sea ice and land”, IEEE Tran. Geosci. Remote Sens., vol. 55, no. 2, pp. 834-843, 2017. DOI: 10.1109/TGRS.2016.2616134.

[10] D. Henry, H. Aubert, T. Veronese, E. Serrano, "Remote estimation of intra-parcel grape quantity from three-dimensional imagery technique using ground-based microwave FMCW radar", IEEE Instrum. Meas. Mag., vol. 20, no. 3, pp. 20-24, 2017. DOI: 10.1109/MIM.2017.7951687.

[11] S. Park, S. Park, "Configuration of an X-band FMCW radar targeted for drone detection", 2017 Int. Symposium on Antennas and Propagation (ISAP 2017), Phuket, 2017, pp. 1-2. DOI: 10.1109/ISANP.2017.8228912.

[12] S. Kim, B. Kim, D. Oh, J. Lee, “An effective pre-processing technique for robust ESPRIT-based single-tone frequency estimation against an I/Q mismatch", Elektronika ir Elektrotechnika, vol. 21, no. 6, pp. 3439, 2015. DOI: 10.5755/j01.eee.21.6.13757.

[13] X. Zhang, L. Xu, D. Xu, "Direction of departure (DOD) and direction of arrival (DOA) estimation in MIMO radar with reduced-dimension MUSIC", IEEE Commun. Lett., vol. 14, no. 12, pp. 1161-1163, 2010. DOI: 10.1109/LCOMM.2010.102610.101581.

[14] X. Zhang, D. Xu, "Low-complexity ESPRIT-based DOA estimation for colocated MIMO radar using reduced-dimension transformation", Electron. Lett., vol. 47, no. 4, pp. 283-284, 2011. DOI: 10.1049/el.2010.3279.

[15] D. Oh, J. Lee, "Low-complexity range-azimuth FMCW radar sensor using joint angle and delay estimation without SVD and EVD", IEEE Sensors J., vol. 15, no. 9, pp. 4799-4811, 2015. DOI: 10.1109/JSEN.2015.2428814.

[16] S. Kim, J. Lee, “A new fine Doppler frequency estimator based on two-sample FFT for pulse Doppler radar", IEICE trans. Commun., vol. 96, no. 6, pp. 1643-1646, 2013. DOI: https://doi.org/10.1587/transcom.E96.B.1643. 